\title{
Projeto AvaTEA: Desenvolvendo um Jogo para a Conscientização do Transtorno do Espectro Autista
}

\author{
Victória O. Gomes ${ }^{1}$, Victor T. Sarinho ${ }^{1}$ \\ ${ }^{1}$ Laboratório de Entretenimento Digital Aplicado - LEnDA \\ Universidade Estadual de Feira de Santana - UEFS \\ Feira de Santana - Bahia - Brasil \\ victoria.oliveiragomes@gmail.com, vsarinho@uefs.br
}

\begin{abstract}
Autistic Spectrum Disorder (ASD) is a neurological disorder that affects the individual development in multiple aspects. In order to achieve a proper inclusion of such individuals in society, it is important to raise awareness among neurotypics about the peculiarities of this disorder. Aiming to contribute to this process, this article presents AvaTEA, a digital game where it is possible to care for and interact with an autistic girl, in order to obtain a greater understanding about these individuals, where, from the monitoring of Ava's routine and some games available, some characteristics common to TEA will be portrayed to the player.
\end{abstract}

Resumo. O Transtorno do Espectro Autista (TEA) constitui uma desordem neurológica que afeta o desenvolvimento do indivíduo em múltiplos aspectos. Para alcançar uma devida inclusão de tais indivíduos na sociedade, é importante conscientizar os neurotípicos acerca das peculiaridades deste transtorno. Almejando contribuir com esse processo, este artigo apresenta o AvaTEA, um jogo digital onde é possível cuidar e interagir com uma menina autista, visando obter uma maior compreensão acerca destes indivíduos, onde, a partir do acompanhamento da rotina de Ava e de alguns games disponíveis, algumas características comuns ao TEA serão retratadas ao jogador.

\section{Introdução}

Os Transtornos Invasivos do Desenvolvimento (TID) são definidos como uma série de condições caracterizadas por deficiências no desenvolvimento de habilidades socias, de comunicação e por um padrão comportamental restrito [Sato et al. 2009]. Dentro desta categoria é possível verificar a presença de diversos transtornos e síndromes, os quais podem variar em aspectos como o perfil da sintomatologia e o grau de acometimento. Eles são agrupados, no entanto, por possuírem em comum a característica relacionada a interrupção precoce no amadurecimento dos processos de socialização [Klin 2006].

Dentre os TID conhecidos, o Transtorno do Espectro Autista (TEA) é um dos que apresenta mais destaque, dado o aumento da incidência do autismo diagnosticado [Whitman 2019]. Tal transtorno consiste em uma deficiência no desenvolvimento de etiologias múltiplas, definido de acordo com fundamentos eminentemente clínicos [Lemos et al. 2014] e caracterizado por "um conjunto heterogêneo de distúrbios da socialização, com início precoce e curso crônico, que possuem um impacto variável em áreas múltiplas e nucleares do desenvolvimento" [Duarte et al. 2016]. 
Quanto mais cedo é obtido o diagnóstico e se dá início as intervenções, maiores as chances de minimizar as características e os impactos provindos do autismo na vida da criança [Volkmar et al. 2014]. Considerando que a família é a principal e primeira instituição com a qual a criança entra em contato com um espaço de socialização [Maia Filho et al. 2016], o posicionamento desta em frente ao autismo possui um papel fundamental no desenvolvimento da criança [Serra 2010].

Assim, buscando obter maiores avanços no quadro da criança, grande parte dos programas de terapia criados para autistas envolve também os familiares, onde, na grande maioria das vezes, seu sucesso depende da continuidade da utilização das técnicas também fora do consultório [Glat 2003]. A maneira como os comportamentos da criança serão abordados e quais práticas serão encorajadas e enfatizadas é de fundamental importância, a fim de que não haja um retrocesso.

Considerando o aumento da incidência de autistas e a necessidade de saber lidar com estes, além da importância de conscientizar os neurotípicos sobre o TEA e, assim, promover uma maior integração desses indivíduos na sociedade, esse artigo apresenta o AvaTEA. Trata-se de um jogo digital onde será possível interagir e cuidar de Ava, um avatar que representa uma menina autista, e, a partir disso, entender um pouco mais sobre como os portadores desse transtorno recebem, interpretam e lidam com os estímulos vindos do mundo exterior. A partir disso, será possível identificar como reagir frente a alguns comportamentos comuns a estas crianças, entendendo o que os desencadeiam.

\section{Metodologia}

A fim de promover um maior entendimento e uma conscientização em neurotípicos acerca dos portadores do TEA, o AvaTEA foi construído visando destacar características e comportamentos apresentados por tais indivíduos. Para tanto, foi necessário fazer o levantamento de comportamentos comuns em portadores do TEA, o que foi baseado na literatura, e, a partir disso, decidiu-se de que maneira eles seriam encaixados no jogo.

Uma das primeiras particularidades encontradas foi a questão da rigidez e a insistência obsessiva na manutenção de uma rotina, por conta da constante necessidade de prever o que acontecerá em sequência no seu dia a dia, e, assim, minimizar a sensação de ansiedade [Sanini et al. 2008]. Para simular isso no jogo, há uma rotina para a personagem, a qual deverá ser seguida pelo jogador. Esta possui horários fixos para atividades específicas e se apresentam logo que o jogo começa.

Visando dar uma maior fluidez ao software e inserir algumas das complicações com as quais são importantes saber lidar, alguns imprevistos ocorrerão durante essa tentativa de cumprir a rotina, levando o jogador a se familiarizar com o comportamento de portadores do TEA frente a frustrações, as quais são frequentemente vistas de maneira extremamente ruim por estes [Sousa et al. 2012].

Além dessas características comportamentais exibidas frente a modificações na rotina, o jogo também busca transmitir a sensação tida pelos neuroatípicos ao receber estímulos que, apesar de parecerem simples para nós, são excessivos para eles. Tais sensações são baseadas em três tipos principais de hipersensibilidade que podem estar presente nestes indivíduos: hipersensibilidade auditiva [Gomes et al. 2004], hipersensibilidade visual [Vila et al. 2009] ou hipersensibilidade tátil [Vila et al. 2009]. 
Assim, ao abrir a cortina pela manhã, por exemplo, o app exibe uma luz branca muito forte em toda a tela, e ao frequentar locais mais movimentados e barulhentos, um som que incomoda consideravelmente é tocado, a fim de proporcionar um desconforto próximo ao que os autistas sentem em tais situações.

Frente a cada um dos acontecimentos adversos do jogo, aparecerão opções textuais juntamente com a descrição do comportamento para que o jogador escolha a melhor maneira, com base na sua concepção, de manejar a situação atual e, a partir disso ele receberá um feedback em relação ao caminho que foi selecionado. Em situações relacionadas somente a sensações, estas serão descritas e a importância de preparar a criança para enfrentá-las será enfatizada.

Para abordar a questão da socialização e da rigidez, alguns minigames serão disponibilizados, o quais serão também a maneira de ganhar moedinhas no jogo para cumprir as atividades necessárias a rotina de Ava. Estes minigames foram selecionados com base em atividades simples que podem ser feitas em casa a fim de promover um avanço em relação as habilidades da criança em relação a aspectos específicos, associando a diversão com o aspecto terapêutico.

Até o momento foram idealizados quatro minigames, os quais foram escolhidos visando trabalhar aspectos relacionados a coordenação motora fina, a estimular o desenvolvimento da atenção compartilhada e a questão da troca de turnos, que são aspectos que frequentemente precisam ser trabalhados em crianças com TEA [Rogers et al. 2015]. O quarto está relacionado a uma atividade feita regularmente por tais indivíduos.

Em relação ao ambiente de desenvolvimento utilizado, optou-se pelo Godot, uma engine para criação de jogos cuja programação é realizada em GDScript, baseada em Python. A partir dele, é possível exportar o jogo para ser executado tanto em dispositivos mobile, que é o maior foco do AvaTEA, como para o PC ou para a Web.

\section{Resultados e Discussão}

A tela inicial confeccionada para o AvaTEA pode ser observada na Figura 1, onde o quarto de Ava é retratado.

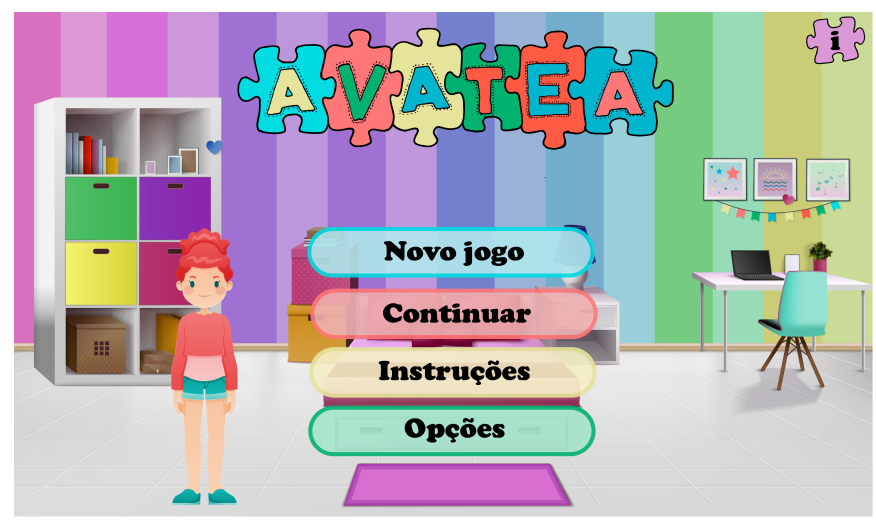

Figure 1. Tela inicial do AvaTEA.

Ao iniciar um novo jogo, você será apresentado a rotina semanal de Ava, como pode ser observado na Figura 2. Seu objetivo principal é fazer com que sua personagem 
cumpra as atividades designadas nos horários e de maneira correta. Nos demais horários, o jogador decidirá quais atividades irá realizar com Ava.

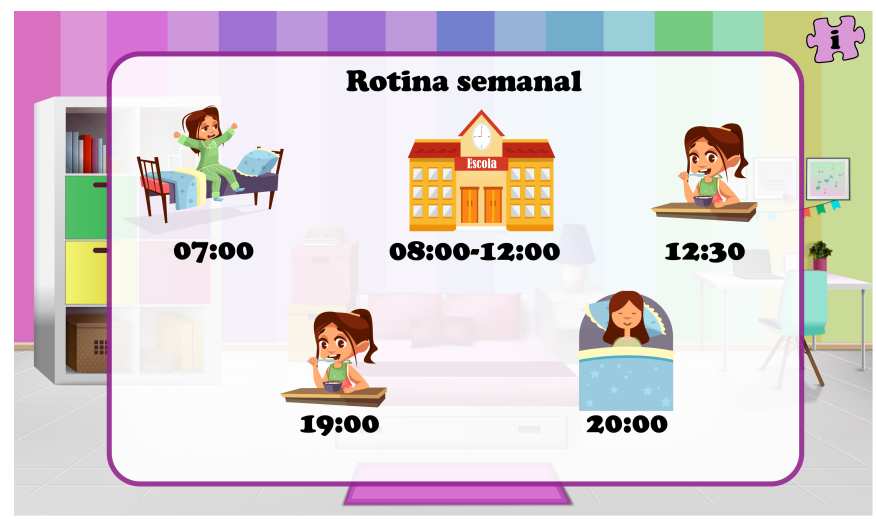

Figure 2. Rotina semanal de Ava.

Para promover uma maior semelhança com a realidade, surgirão certos contratempos e conflitos durante o jogo que impedirão o cumprimento fiel dessa rotina, e, a partir do aparecimento destes, algumas opções de como lidar com as reações de Ava serão disponibilizadas, como pode ser observado no exemplo exibido na Figura 3.

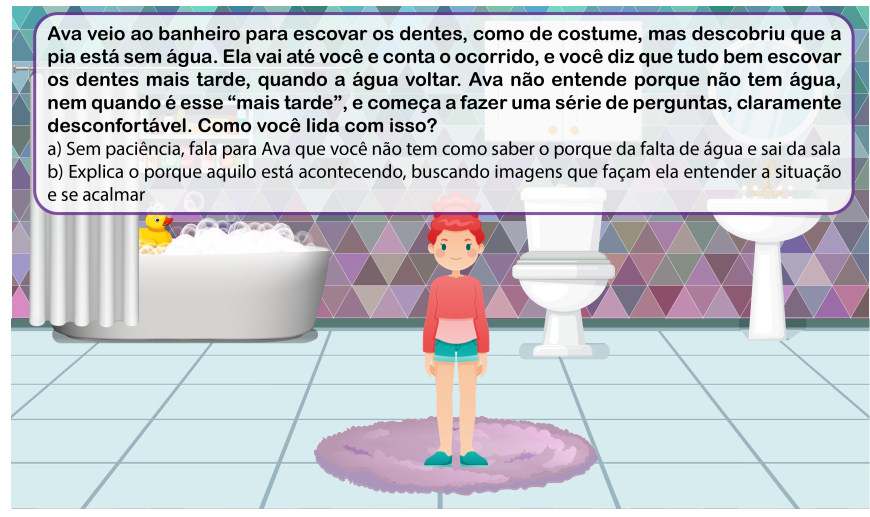

Figure 3. Exemplo de escolha disponibilizada para o jogador.

Em relação aos minigames disponíveis no jogo, no primeiro deles, o jogador será levado a uma tela com quatro garrafas de vidro, e terá a missão de colocar as bolinhas presentes em uma cesta dentro dessas garrafas, sem que elas caiam no chão, como pode ser visto na Figura 4. Para "simular" as dificuldades relativas a coordenação motora de portadores do TEA, o cenário estará em constante movimento, algo como um balanço, fazendo com que fique mais difícil acertar. Tal jogo foi escolhido com base nos déficits das habilidades motoras que são observados em portadores do TEA [Association et al. 2013], e que precisam ser trabalhados como base para alcançar outras habilidades como, por exemplo, aprender a escrever.

No segundo minigame, o jogador irá encontrar o rosto de Ava. Utilizando o conceito de atenção compartilhada, o que visa fazer com que a criança olhe diretamente para você, o olhos de Ava estarão constantemente trocando de direção e, quando estiverem direcionados para o jogador, este deverá clicar na tela, gerando assim um reforço positivo. 


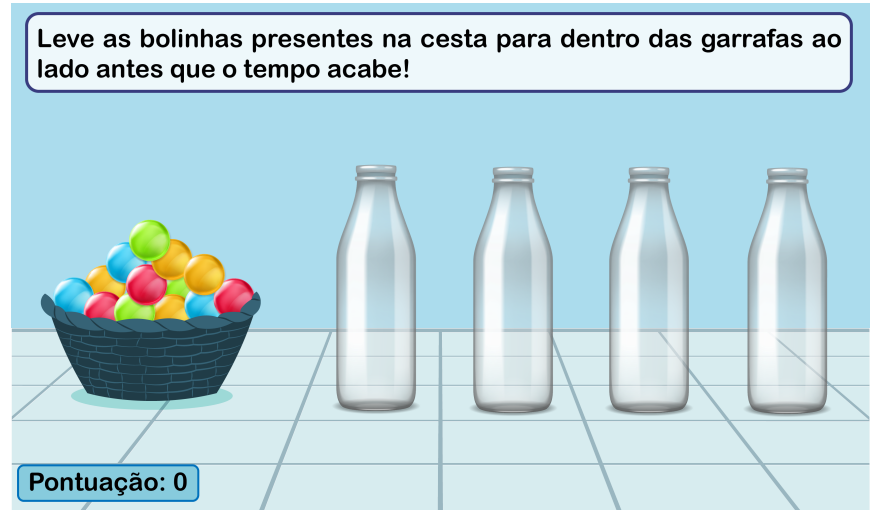

Figure 4. Minigame 1 disponível no AvaTEA.

Como a habilidade relacionada a troca de turnos consiste em saber que existem momentos que você deverá ter a atenção e, em outros, você deverá aguardar seu parceiro, no terceiro game temos a simulação de um jogo da memória entre Ava e outro oponente. Seu objetivo será fazer com que Ava aguarde a sua vez de jogar, a qual irá demorar cada vez mais para chegar. Para isso, sempre que Ava tentar ir em direção ao jogo, você deverá clicar nela, emitindo assim uma mensagem explicando que ela deve aguardar pacientemente seu oponente jogar.

No quarto minigame, teremos a simulação de uma atividade cuja realização é frequentemente observada em autistas: a questão da organização por cores, a qual está relacionada a um comportamento esteriotipado [da Silva 2020]. O jogador irá visualizar cinco tubos, sendo que parte deles estará preenchido com bolinhas coloridas. Para ganhar, é necessário que você ajude Ava a separá-los por cores, limitando cada tubo a uma cor.

\section{Conclusões e Trabalhos Futuros}

O presente artigo detalhou os parâmetros seguidos para o projeto do AvaTEA, um jogo voltado para a conscientização de neurotípicos acerca do Transtorno do Espectro Autista (TEA). Tal jogo visa maximizar a integração de tais indivíduos na sociedade, o que é viável a partir da compreensão das suas peculiaridades por parte dos demais.

É importante salientar, entretanto, que cada indivíduo autista possui características e peculiaridades únicas. Sendo assim, apesar de cada uma das situações exemplificadas no jogo ser embasada na literatura, não é garantido que todos os portadores do TEA irão reagir da maneira retratada no jogo quando receberem estímulos semelhantes. Apesar disso, a escolha das situações abordadas foi feita com base no comportamento apresentado pela grande maioria, visando obter tanta semelhança a realidade quanto possível.

Como trabalhos futuros, almeja-se o desenvolvimento de mais minigames, além de promover uma maior variação na rotina disponibilizada e uma maior quantidade de interações com o personagem, evidenciando cada vez mais características presentes no TEA. Isso, no entanto, será feito após a realização de uma validação com o público alvo (neurotípicos), para que identifiquemos melhorias a serem feitas no estado atual do jogo antes de prosseguir para a adição de novas funcionalidades. Além disso, almeja-se que um profissional na área do TEA participe dessa avaliação, a fim confirmar que os critérios selecionados foram utilizados corretamente. 


\section{References}

Association, A. P. et al. (2013). Diagnostic and statistical manual of mental disorders (DSM-5®). American Psychiatric Pub.

da Silva, A. L. (2020). Comportamento estereotipado no transtorno do espectro autista. Desafios-Revista Interdisciplinar da Universidade Federal do Tocantins, 7(1):96-108.

Duarte, C. P., Schwartzman, J. S., Matsumoto, M. S., and Brunoni, D. (2016). Diagnóstico e intervenção precoce no transtorno do espectro do autismo: Relato de um caso. Caminha, VL, Huguenin, J., Assis, LM \& Alves, PP (Org.), pages 45-56.

Glat, R. (2003). Convivendo com filhos especiais o olhar paterno, volume 5. 7Letras.

Gomes, E., Rotta, N. T., Pedroso, F. S., Sleifer, P., and Danesi, M. C. (2004). Hipersensibilidade auditiva em crianças e adolescentes com transtorno do espectro autista. Arquivos de Neuro-Psiquiatria, 62(3B):797-801.

Klin, A. (2006). Autismo e síndrome de asperger: uma visão geral. Brazilian Journal of Psychiatry, 28:s3-s11.

Lemos, E., Salomão, N. M. R., and Agripino-Ramos, C. S. (2014). Inclusão de crianças autistas: um estudo sobre interações sociais no contexto escolar. Revista Brasileira de Educação Especial, 20(1):117-130.

Maia Filho, A. L. M., Amanda, L. A. N. M. N., Nogueira, L., Silva, K. C. O., and Santiago, R. F. (2016). A importância da família no cuidado da criança autista/the importance of the family in the care of autist children. Saúde em Foco, 3(1):66-83.

Rogers, S., Dawson, G., and Vismara, L. (2015). Autismo - Compreender E Agir Em Familia. LIDEL.

Sanini, C., Ferreira, G. D., Souza, T. S., and Bosa, C. A. (2008). Comportamentos indicativos de apego em crianças com autismo. Psicologia: Reflexão e Crítica, 21(1):60-65.

Sato, F. P., Paula, C. S., Lowenthal, R., Nakano, E. Y., Brunoni, D., Schwartzman, J. S., and Mercadante, M. T. (2009). Instrumento para rastreamento dos casos de transtorno invasivo do desenvolvimento: estudo preliminar de validação. Brazilian Journal of Psychiatry, 31(1):30-33.

Serra, D. (2010). Autismo, família e inclusão. Polêm! ca, 9(1):40-56.

Sousa, F. R. M., Costa, E. A. B., and de Castro, T. H. C. (2012). Worldtour: Software para suporte no ensino de crianças autistas. In Brazilian Symposium on Computers in Education (Simpósio Brasileiro de Informática na Educação-SBIE), volume 23.

Vila, C., Diogo, S., and Sequeira, S. (2009). Autismo e síndrome de asperger. Revista científica.

Volkmar, F., Siegel, M., Woodbury-Smith, M., King, B., McCracken, J., State, M., et al. (2014). Practice parameter for the assessment and treatment of children and adolescents with autism spectrum disorder. Journal of the American Academy of Child \& Adolescent Psychiatry, 53(2):237-257.

Whitman, T. L. (2019). O desenvolvimento do autismo. M. Books Editora. 